from 2015 to 2017. We fitted linear mixed models for Rheumatoid Arthritis Disease Activity Index (RADAI), Fatigue visual analog scale (VAS), and Pain VAS scores separately without/with adjusting for age, sex, sociodemographic factors, body mass index, RA duration, physical function (Modified Health Assessment Questionnaire [mHAQ]), smoking, depression and other comorbidities at baseline. Models include the outcome at three years (2015-2017) as the dependent variables, an indicator variable representing the year of measurement (2015-17), PA level at baseline, their interactions, and baseline characteristics as covariates. Models include random effects for subjects to account for correlations among within-subject repeated measures. Missing values were imputed using multiple imputation methods. The analyses were conducted using $\mathrm{R} 3.5$.

Results: Of the 169 patients who responded to the 2015 survey, 29.6\%, $42.0 \%$, and $28.4 \%$ had low, medium, and high levels of PA in 2015, respectively. There were no significant differences in RADAI ( $p=0.67)$, Fatigue $(p=0.78)$, and Pain $(p=0.98)$ at baseline (Figure 1). The low PA group experienced significant worsening of disease activity outcomes over time, including yearly increases of 0.31 in RADAI $(p=0.007), 0.41$ in Fatigue $(p=0.007)$, and 0.43 in Pain $(p=0.007)$. Those in the medium and high PA groups at baseline experienced either a decrease or no change in their disease activity outcomes over time. The interaction terms for PA level and year show that the low PA group had significantly different time trends for RADAI, Fatigue, and Pain compared to the medium and high PA groups (all $p$ values $<0.05$ ). The results remain robust after adjusting for age, sex, and other covariates.

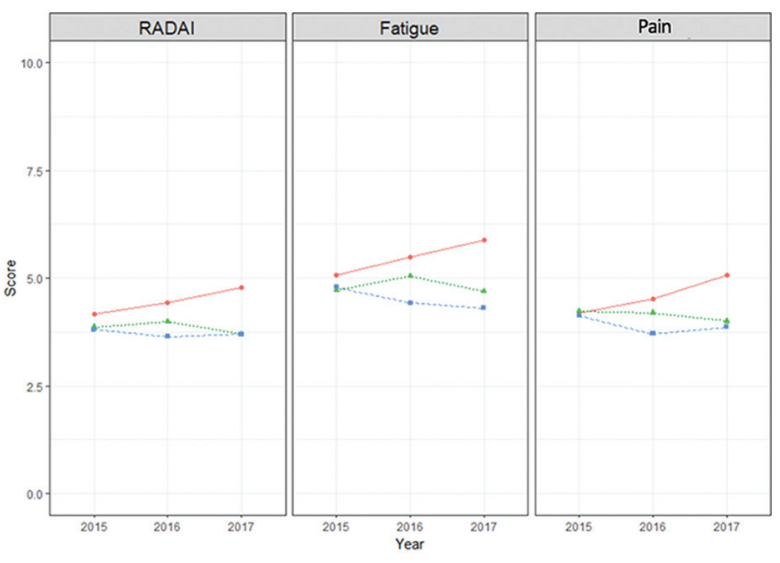

Physical Activity $\rightarrow$ Low $-\cdots \cdot$. Medium -- High

Figure 1. Time Trends for Mean RADAI, Fatigue, and Pain by Baseline Physical Activity Level

Table 1. Linear Mixed Model without/with Adjusting for Baseline Characteristics

\begin{tabular}{|c|c|c|c|c|c|c|}
\hline \multirow{2}{*}{$\begin{array}{l}\text { Model } \\
\text { Covariates }\end{array}$} & \multicolumn{2}{|l|}{ RADAI } & \multicolumn{2}{|l|}{ Fatigue } & \multicolumn{2}{|l|}{ Pain } \\
\hline & Coef. $[95 \% \mathrm{Cl}]$ & $p$ & Coef. $[95 \% \mathrm{Cl}]$ & p & Coef. $[95 \% \mathrm{Cl}]$ & p \\
\hline & & A. Una & id Linear Mixed Mod & & & \\
\hline \multicolumn{7}{|l|}{ Physical Activity (PA) } \\
\hline Low & ref & & ref & & ref & \\
\hline Medium & $-0.24[-0.99,0.52]$ & 0.542 & $-0.24[-1.26,0.77]$ & 0.638 & $0.08[-0.87,1.04]$ & 0.865 \\
\hline High & $-0.39[-1.22,0.44]$ & 0.357 & $-0.33[-1.44,0.78]$ & 0.558 & $-0.13[-1.17,0.92]$ & 0.811 \\
\hline Year (Low PA Group) & $0.31[0.07,0.54]$ & 0.012 & $0.41[0.1,0.72]$ & 0.009 & $0.43[0.11,0.75]$ & 0.009 \\
\hline \multicolumn{7}{|l|}{$\begin{array}{l}\text { Physical ActivityyYYe. } \\
\text { Mediumyer* }\end{array}$} \\
\hline $\begin{array}{l}\text { Medium\#YYear* } \\
\text { HightIYear"* }\end{array}$ & $\begin{array}{l}-0.36[-0.67,-0.05] \\
-0.35[-0.69,-0.01]\end{array}$ & $\begin{array}{l}0.025 \\
0.048\end{array}$ & $\begin{array}{l}-0.44[-0.84,-0.03] \\
-0.68[-1.12,-0.24]\end{array}$ & $\begin{array}{l}0.036 \\
0.003\end{array}$ & $\begin{array}{l}-0.5[-0.92,-0.08] \\
-0.54[-1.01,-0.08]\end{array}$ & 0.021 \\
\hline \multicolumn{7}{|c|}{ 8. Adjusted Linear Mixed Model } \\
\hline \multicolumn{7}{|l|}{ Physical Activity (PA) } \\
\hline Low & ref & & ref & & ref & \\
\hline Medium & $0.54[-0.22,1.29]$ & 0.162 & $0.25[-0.55,1.06]$ & 0.537 & $0.54[-0.22,1.29]$ & 0.162 \\
\hline High & $0.94[0.11,1.77]$ & 0.027 & $0.74[-0.15,1.63]$ & 0.105 & $0.94[0.11,1.77]$ & 0.027 \\
\hline $\begin{array}{l}\text { Year (Low PA Group) } \\
\text { Physical Activity\#Year }\end{array}$ & $0.44[0.12,0.77]$ & 0.007 & $0.43[0.12,0.74]$ & 0.007 & $0.44[0.12,0.77]$ & 0.007 \\
\hline MediumtYear* & $-0.51[-0.94,-0.08]$ & 0.019 & $-0.46[-0.86,-0.05]$ & 0.028 & $-0.51[-0.94,-0.08]$ & 0.019 \\
\hline HightiYear"* & $-0.56[-1.03,-0.1]$ & & $-0.7[-1.14,-0.26]$ & & $-0.56[-1.03,-0.1]$ & 0.017 \\
\hline
\end{tabular}

Conclusion: Across RADAI, Fatigue VAS, and Pain VAS scores, RA patients with a low PA level demonstrated a significant increase in disease activity over the span of three years. The trends for both the high and medium PA groups differ significantly from that of the low PA group, showing small, overall decreases in disease activity over time. These results add to the accumulating evidence that physical activity may reduce disease activity and is an essential aspect of $R A$ management. Acknowledgement: Funded by the CIHR

Disclosure of Interests: Kiera Lee-Pii: None declared, Hui Xie: None declared, Yufei Zheng: None declared, Linda Li: None declared, Diane Lacaille Grant/research support from: Bristol-Myers Squibb and Eli Lilly Canada
DOI: 10.1136/annrheumdis-2019-eular.2515

\section{SAT0098 ANTIBODIES AGAINST CITRULLINATED ANTIGENS ARE ASSOCIATED WITH TREATMENT RESPONSE IN ACPA- NEGATIVE RHEUMATOID ARTHRITIS PATIENTS}

Stephanie Ling ${ }^{1,2}$, Nisha Nair ${ }^{1}$, Darren Plant ${ }^{1}$, Hans-Dieter Zucht ${ }^{3}$, Petra Budde ${ }^{3}$, Peter Schulz-Knappe ${ }^{3}$, Matura Consortium ${ }^{4}$, Anne Barton ${ }^{1,2}$. ${ }^{1}$ Arthritis Research UK Centre for Genetics and Genomics, The University of Manchester, Manchester, United Kingdom; ${ }^{2}$ NIHR Manchester BRC, Manchester Foundation Trust, Manchester, United Kingdom; ${ }^{3}$ Protagen AG, Dortmund, Germany, ${ }^{4}$ MATURA, Multi-centre, United Kingdom

Background: RA patients who are ACPA-positive $\left(\mathrm{ACPA}^{+}\right)$are known to have worse prognosis. Less is known regarding predictors of treatment outcomes in ACPA-negative (ACPA') patients.

Objectives: We investigated whether autoantibodies captured on a custom array were associated with response to therapy in ACPA $^{-}$RA patients. Methods: RA patients were recruited to either the Biologics in RA Genet ics and Genomics Study Syndicate (BRAGGSS, starting adalimumab, established disease) or the RA Medication Study (RAMS, starting methotrexate, early disease). Serum samples were collected at pre-treatment and $3 / 6$ months in BRAGGSS/RAMS, respectively. Treatment groups were pooled for analysis. ACPA was measured using a commercially available ELISA (Axis-Shield Diagnostics Ltd, Dundee, UK). Pre-treatment RA and healthy blood donor control $(\mathrm{HC})$ serum samples were incubated on a bead-based assay (Luminex FlexMap 3D) containing 376 human protein antigens associated with autoimmune disease (39 in citrullinated (cit) form) to detect autoantibodies. Median fluorescence intensity (MFI) values were calculated for each autoantibody for RA and $\mathrm{HC}$, then normalised and log2-transformed. The $95^{\text {th }}$ percentile for each autoantibody in $\mathrm{HC}$ was used to determine whether an RA sample was positive/negative for that autoantibody. Proteins with $<10 \%$ frequency in RA patients were excluded from analysis. Linear regression was used to determine autoantibodies differing in $\mathrm{MFI}$ between $\mathrm{RA}$ and $\mathrm{HC}$; $\mathrm{p}$-values were adjusted using the Benjamini-Hochberg correction. Significant autoantibodies (adjusted $\mathrm{p}<0.05$ ) were tested for associations with treatment outcomes in RA patients only using: (i) linear regression for improvement in DAS28; (ii) logistic regression for good/poor vs all EULAR response. All regression was adjusted for age, gender, disease duration and baseline DAS28. Subanalysis was carried out in a subset of ACPApatients.

Results: 168 patients with RA were included in analysis (mean age 59.6 years, mean disease duration 14.3 years, 126 (75\%) female patients, 90 (53.6\%) $\mathrm{ACPA}^{+}$patients). 34 autoantibodies were differentially expressed in RA patients, only one of which (TNF ligand superfamily member 13 , TNFSF13) was lower than in HC. 29/34 autoantigens were in cit-form. In multivariate models of all differentially expressed autoantibodies, heterogeneous nuclear ribonucleoprotein A1 (HNRNPA1) was significantly associated with EULAR response (coefficient (coef) $0.7,95 \% \mathrm{Cl} 0.1-1.3$ ), and cit-vimentin (VIM) was significantly associated with poor EULAR response $\left(\mathrm{OR}_{\mathrm{adj}} 4.2,95 \% \mathrm{Cl} 1.1-18.3\right)$ and reduced odds of good EULAR response $\left(\mathrm{OR}_{\mathrm{adj}} 0.2,95 \% \mathrm{Cl} 0.1-0.8\right)$. ACPA remained the best predictor of treatment response. In a subanalysis of the 78 ACPA-patients, citcleavage and polyadenylation specificity factor subunit 6 (CPSF6) was significantly associated with worse DAS28 (coef (-1.8), 95\% Cl (-3.6)-($0.1)$ ). cit-DnaJ homolog subfamily B member 1 (DNAJB1) was significantly associated with DAS28 improvement (coef 2.2, 95\% Cl 0.6-3.8). No autoantibody was associated with EULAR response.

Conclusion: A subset of ACPA-patients have ACPA fine specificities not seen by a commercial assay. Larger ACPA profiling may provide additional information on treatment response. This requires validation with larger sample sizes and replication in an independent cohort.

\section{REFERENCE}

[1] Ann Rheum Dis 2013;72:844. 2. Arthritis Res Ther 2016;18:235.

Disclosure of Interests: Stephanie Ling: None declared, Nisha Nair: None declared, Darren Plant: None declared, Hans-Dieter Zucht Employee of: Hans-Dieter Zucht is an employee of Protagen AG, Petra Budde Employee of: Petra Budde is an employee of Protagen AG, Peter Schulz-Knappe Shareholder of: Peter Schulz-Knappe is a shareholder of Protagen AG, Consultant for: Peter Schulz-Knappe is a consultant to Protagen AG, Employee of: Peter Schulz-Knappe was an employee of Prota gen AG, MATURA Consortium: None declared, Anne Barton: None declared

DOI: 10.1136/annrheumdis-2019-eular.3249 